\title{
Sturm theorem for the generalized Frank matrix ${ }^{*}$
}

\author{
Efruz Özlem Mersin ${ }^{\dagger 1}$ (D), Mustafa Bahşi ${ }^{2}$ \\ ${ }^{1}$ Department of Mathematics, Aksaray University, Aksaray, Turkey \\ ${ }^{2}$ Department of Mathematics and Science Education, Aksaray University, Aksaray, Turkey
}

\begin{abstract}
One of the popular test matrices for eigenvalue routines is the Frank matrix due to its wellconditioned and poorly conditioned eigenvalues. All the eigenvalues of the Frank matrix are real, positive and different. Sturm Theorem is a very useful tool for computing the eigenvalues of tridiagonal symmetric matrices. In this paper, we apply Sturm Theorem to the generalized Frank matrix which is a special form of the Hessenberg matrix and examine its eigenvalues by using Sturm property. Moreover, we illustrate our results with an example.
\end{abstract}

Mathematics Subject Classification (2020). 15A18, 15B99

Keywords. Sturm sequence, characteristic polynomial, eigenvalue, Frank matrix.

\section{Introduction}

The problem of finding zeros of a polynomial with real coefficients

$$
p(x)=a_{n} x^{n}+a_{n-1} x^{n-1}+\cdots+a_{0}
$$

has been very important for the applications of algebra. Unfortunately for $n \geq 5$, we do not have an exact formula for the zeros of the polynomial (1.1). We only have some iterative methods such as Newton's formula [7] and also some bounds such as Cauchy's bound [1]. Moreover, while Descartes' rule of sign [1] and Budan Fourier Theorem [1] give us upper bounds for the number of zeros in an interval, Sturm Theorem gives the exact number of zeros in an interval for any polynomial without multiple zeros. Sturm Theorem, first appeared in 1829, has been known by means of Sturm's studies [13-15]. He solved the problem of the number of zeros in an interval, by using the number of sign changes in Sturm sequence

$$
p_{0}(x), p_{1}(x), \ldots, p_{n}(x) .
$$

The simplest way to construct a Sturm sequence is to apply the Euclidean Algorithm to $p(x)$ and its derivative $[1,7,10]$. Many versions and analogues of Sturm sequences and Sturm Theorem have been studied in the literature $[4,6,10,12]$. We use the following analogy in this study.

\footnotetext{
${ }^{*}$ This article is a part of the doctoral thesis being prepared by Efruz Özlem Mersin.

†Corresponding Author.

Email addresses: efruzmersin@gmail.com (E.Ö. Mersin), mhvbahsi@yahoo.com (M. Bahşi)

Received: 24.07.2020; Accepted: 12.02.2021
} 
Definition $1.1([4])$. Let $p_{0}(x), p_{1}(x), \ldots, p_{n}(x)$ be continuous functions on an interval $(a, b)$ (the possibilities $a=-\infty, b=\infty$ are included). We say that the sequence $p_{0}(x), p_{1}(x), \ldots, p_{n}(x)$ has the Sturm property if:

(a) $p_{0}(x)$ has no zeros in $(a, b)$,

(b) For $1 \leq i \leq n$, the set of zeros of $p_{i}(x)$ is discrete,

(c) For $1 \leq i \leq n-1$, if $p_{i}\left(x_{0}\right)=0$, then $p_{i-1}\left(x_{0}\right) p_{i+1}\left(x_{0}\right)<0$,

(d) For $1 \leq i \leq n$, if $p_{i}\left(x_{0}\right)=0$, then for sufficiently small $\varepsilon_{1}, \varepsilon_{2}>0$, $p_{i-1}\left(x_{0}\right)\left[p_{i}\left(x_{0}+\varepsilon_{2}\right)-p_{i}\left(x_{0}-\varepsilon_{1}\right)\right]<0$.

Theorem $1.2([4])$. Suppose that the sequence $p_{0}(x), p_{1}(x), \ldots, p_{n}(x)$ has the Sturm property on $(a, b)$ and let $\alpha<\beta$ be numbers in $(a, b)$, then $p_{n}(x)$ has exactly $c(\beta)-c(\alpha)$ different zeros in the interval $(\alpha, \beta)$, where $c(\alpha)$ denotes the number of changes in sign of consecutive members of the sequence $p_{0}(\alpha), p_{1}(\alpha), \ldots, p_{n}(\alpha)$.

Theorem $1.3([4])$. Suppose that $p_{0}(x), p_{1}(x), \ldots, p_{n}(x)$ is a Sturm sequence, then for $1 \leq i \leq n$, the zeros of $p_{i}(x)$ and $p_{i-1}(x)$ are interlaced.

Sturm Theorem is a very useful tool for computing the eigenvalues of tridiagonal symmetric matrices $[4,11,12,17]$. For example, Greenberg [4] has defined symmetric tridiagonal matrices of the form

$$
[A(\lambda)]_{i, j=1}^{n}=\left\{\begin{array}{cl}
b_{i-1}+b_{i}-a_{i}, & \text { if } i=j \\
b_{\min (i, j)}, & \text { if }|i-j|=1 \\
0, & \text { otherwise }
\end{array}\right.
$$

where $a_{i}=a_{i}(\lambda)$ and $b_{j}=b_{j}(\lambda)$ are continuous functions on $(a, b)$ and has applied Sturm theory to nonlinear eigenvalue problem of the form $\operatorname{det}[A(\lambda)]$ using the recurrence relation

$$
S_{i+1}=\left(b_{i}+b_{i+1}-a_{i+1}\right) S_{i}-b_{i}^{2} S_{i-1}
$$

for $1 \leq i \leq n-1$, where $S_{i}$ is the $i$ th principal minor of $[A(\lambda)]$, and $S_{0}=1$. In the present paper, we apply Sturm theory to the generalized Frank matrices. For more information related to the axiomatic development of Sturm sequences, we refer to [4].

One of the popular test matrices for eigenvalue routines is the Frank matrix due to its well-conditioned and poorly conditioned eigenvalues $[2,16]$. Frank [3] defined the matrix

$$
F_{n}=\left\{\begin{array}{cc}
n+1-\max (i, j), & i>j-2 \\
0, & \text { otherwise }
\end{array}=\left[\begin{array}{cccccc}
n & n-1 & 0 & \cdots & 0 & 0 \\
n-1 & n-1 & n-2 & \cdots & 0 & 0 \\
n-2 & n-2 & n-2 & \cdots & 0 & 0 \\
\vdots & \vdots & \vdots & \ddots & \vdots & \vdots \\
2 & 2 & 2 & \cdots & 2 & 1 \\
1 & 1 & 1 & \cdots & 1 & 1
\end{array}\right],\right.
$$

which is called the Frank matrix $[5,16]$. The characteristic polynomial $\chi_{n}(\lambda)$ of $F_{n}$ has the recurrence relation [5]:

$$
\begin{gathered}
\chi_{n}(\lambda)=(1-\lambda) \chi_{n-1}(\lambda)-(n-1) \lambda \chi_{n-2}(\lambda), \quad \text { for } n \geq 3 \\
\chi_{1}(\lambda)=1-\lambda \text { and } \chi_{2}(\lambda)=1-3 \lambda+\lambda^{2} .
\end{gathered}
$$

The Frank matrix is also a Max matrix. Kılıç and Arıkan [8] studied the general MaxMin matrices and stated that how one may obtain some algebraic properties of the Frank matrix by using the Max-Min matrices.

As a result of Sturm Theorem, all the eigenvalues of the matrix $F_{n}$ are real and positive [5]. Consider the $n \times n$ matrix associated with a finite sequence $a=\left(a_{1}, a_{2}, \ldots, a_{n}\right)$, 


$$
\left[F_{a}\right]_{n}=\left\{\begin{array}{cc}
a_{n+1-\max (i, j)}, & i>j-2 \\
0, & \text { otherwise }
\end{array}=\left[\begin{array}{cccccc}
a_{n} & a_{n-1} & 0 & \cdots & 0 & 0 \\
a_{n-1} & a_{n-1} & a_{n-2} & \cdots & 0 & 0 \\
a_{n-2} & a_{n-2} & a_{n-2} & \cdots & 0 & 0 \\
\vdots & \vdots & \vdots & \ddots & \vdots & \vdots \\
a_{2} & a_{2} & a_{2} & \cdots & a_{2} & a_{1} \\
a_{1} & a_{1} & a_{1} & \cdots & a_{1} & a_{1}
\end{array}\right],\right.
$$

where $a_{i}$ 's are real numbers. The matrix $\left[F_{a}\right]_{n}$ is a lower Hessenberg matrix and reduces to the matrix $F_{n}$ for $a_{i}=i$. Therefore, $\left[F_{a}\right]_{n}$ is called as the generalized Frank matrix [9].

Now, by the motivation of the studies on tridiagonal symmetric matrices and Sturm Theorem we have some questions: Does the sequence of characteristic polynomials of $\left[F_{a}\right]_{i \leq n}$ form a Sturm sequence? Can we determine the number of eigenvalues of $\left[F_{a}\right]_{n}$ in any interval? Can we establish some bounds for the eigenvalues of $\left[F_{a}\right]_{n}$ ? In the present paper, we seek to answer these questions.

Throughout the paper, to facilitate the readability we use the notations $P_{i}(\infty)$ and $w_{i}(\infty)$ rather than $\lim _{\lambda \rightarrow \infty} P(\lambda)$ and $\lim _{\lambda \rightarrow \infty} w(\lambda)$, respectively.

\section{Main results}

Lemma 2.1. Let $P_{n}(\lambda)$ be the characteristic polynomial of the matrix $\left[F_{a}\right]_{n}$ and $n \geq 2$. Then, $P_{n}(\lambda)$ satisfies

$$
P_{n}(\lambda)=\left(a_{n}-a_{n-1}-\lambda\right) P_{n-1}(\lambda)-a_{n-1} \lambda P_{n-2}(\lambda),
$$

with initials $P_{0}(\lambda)=1$ and $P_{1}(\lambda)=a_{1}-\lambda$.

Proof. The characteristic polynomial of $\left[F_{a}\right]_{n}$ is

$$
P_{n}(\lambda)=\operatorname{det}\left(\left[F_{a}\right]_{n}-\lambda I\right)=\left|\begin{array}{cccccc}
a_{n}-\lambda & a_{n-1} & 0 & \cdots & 0 & 0 \\
a_{n-1} & a_{n-1}-\lambda & a_{n-2} & \cdots & 0 & 0 \\
a_{n-2} & a_{n-2} & a_{n-2}-\lambda & \cdots & 0 & 0 \\
\vdots & \vdots & \vdots & \ddots & \vdots & \vdots \\
a_{2} & a_{2} & a_{2} & \cdots & a_{2}-\lambda & a_{1} \\
a_{1} & a_{1} & a_{1} & \cdots & a_{1} & a_{1}-\lambda
\end{array}\right| .
$$

Subtracting $(i+1)$ th column from the $i$ th column for $i=1,2, \ldots, n-1$, respectively, we get

$$
P_{n}(\lambda)=\left|\begin{array}{cccccc}
a_{n}-a_{n-1}-\lambda & a_{n-1} & 0 & \cdots & 0 & 0 \\
\lambda & a_{n-1}-a_{n-2}-\lambda & a_{n-2} & \cdots & 0 & 0 \\
0 & \lambda & a_{n-2}-a_{n-3}-\lambda & \cdots & 0 & 0 \\
\vdots & \vdots & \vdots & \ddots & \vdots & \vdots \\
0 & 0 & 0 & \cdots & a_{2}-a_{1}-\lambda & a_{1} \\
0 & 0 & 0 & \cdots & \lambda & a_{1}-\lambda
\end{array}\right| .
$$

By expanding this determinant by the first row and after by the first column, for $n \geq 2$ we have

$$
P_{n}(\lambda)=\left(a_{n}-a_{n-1}-\lambda\right) P_{n-1}(\lambda)-a_{n-1} \lambda P_{n-2}(\lambda),
$$

with initials $P_{0}(\lambda)=1$ and $P_{1}(\lambda)=a_{1}-\lambda$.

Now, we have the sequence

$$
P_{0}(\lambda)=1, P_{1}(\lambda), P_{2}(\lambda), \ldots, P_{n-1}(\lambda), P_{n}(\lambda)
$$

formed by the characteristic polynomials of $\left[F_{a}\right]_{i \leq n}$. 
Lemma 2.2. Suppose that the real sequence $\left\{a_{i}\right\}$ is positive and strictly increasing, then

(a) For $1 \leq i \leq n, P_{i}(\lambda)$ does not vanish for $\lambda=0$. That is, zero is not an eigenvalue of $\left[F_{a}\right]_{i}$, for $1 \leq i \leq n$,

(b) Two consecutive terms $P_{i}(\lambda), P_{i+1}(\lambda)$ can not have a common zero, for $1 \leq i \leq n-1$.

Proof. (a) From the recurrence relation (2.1) and the equality $P_{1}(0)=a_{1}$, we get

$$
\begin{aligned}
P_{i}(0) & =\left(a_{i}-a_{i-1}\right) P_{i-1}(0) \\
& =\left(a_{i}-a_{i-1}\right)\left(a_{i-1}-a_{i-2}\right) \ldots\left(a_{2}-a_{1}\right) a_{1} .
\end{aligned}
$$

Since $a_{i}$ is a positive real number and the sequence $\left\{a_{i}\right\}$ is strictly increasing, we have

$$
P_{i}(0) \neq 0,
$$

for $1 \leq i \leq n$.

(b) Assume that $P_{i+1}\left(\lambda_{0}\right)=P_{i}\left(\lambda_{0}\right)=0$ for $1 \leq i \leq n-1$, then from the recurrence relation (2.1), we get

$$
P_{i-1}\left(\lambda_{0}\right)=P_{i-2}\left(\lambda_{0}\right)=\ldots=P_{0}\left(\lambda_{0}\right)=0,
$$

which contradicts $P_{0}(\lambda)=1$. Thus, two consecutive terms $P_{i}(\lambda), P_{i+1}(\lambda)$ can not have a common zero, for $1 \leq i \leq n-1$.

Lemma 2.3. Suppose that

(1) $a_{i}$ is a positive real number and the sequence $\left\{a_{i}\right\}$ is strictly increasing,

(2) $I_{1} \subset(0, \infty)$ is any interval containing no zeros of $P_{i-1}(\lambda)$, for $1 \leq i \leq n$,

(3) $I_{2} \subset(0, \infty)$ is any interval containing no zeros of $P_{i}(\lambda)$, for $1 \leq i \leq n$,

(4) $I_{3}=(t, \infty)$ is any interval containing no zeros of all $P_{i}(\lambda)$, for $1 \leq i \leq n$.

Then,

(a) $\frac{P_{i}(\lambda)}{P_{i-1}(\lambda)}$ is strictly decreasing on $I_{1}$,

(b) $\frac{\lambda P_{i-1}(\lambda)}{P_{i}(\lambda)}$ is strictly increasing on $I_{2}$,

(c) $(-1)^{i-2} P_{i}(\lambda)<(-1)^{i-1} \lambda P_{i-1}(\lambda)$ for $\lambda \in I_{3}$.

Proof. We will prove that (a) and (b) are valid by induction method on $i$ simultaneously. For $i=1$, it is clear that

$$
\frac{P_{1}(\lambda)}{P_{0}(\lambda)}=\frac{a_{1}-\lambda}{1}=a_{1}-\lambda
$$

is strictly decreasing on every interval and

$$
\frac{\lambda P_{0}(\lambda)}{P_{1}(\lambda)}=\frac{\lambda}{a_{1}-\lambda}=\frac{1}{\frac{a_{1}}{\lambda}-1}
$$

is strictly increasing on any interval that does not contain $a_{1}$ which is unique zero of $P_{1}(\lambda)$. Assume that (a) and (b) are valid for $i \leq k$, we must show that $(a)$ and $(b)$ are valid for $k+1 \leq n$.

(a) Let $J \subset(0, \infty)$ be an interval that has no zeros of $P_{k}(\lambda)$ and $P_{k-1}(\lambda)$. From the recurrence relation $(2.1)$, we have

$$
\frac{P_{k+1}(\lambda)}{P_{k}(\lambda)}=\left(a_{k+1}-a_{k}-\lambda\right)-a_{k} \lambda \frac{P_{k-1}(\lambda)}{P_{k}(\lambda)} .
$$


Since $a_{k+1}-a_{k}-\lambda$ is strictly decreasing and from the assumption (for $i \leq k$ ) $-a_{k} \lambda \frac{P_{k-1}(\lambda)}{P_{k}(\lambda)}$ is strictly decreasing for $a_{k}>0$ in $J$, we have $\frac{P_{k+1}(\lambda)}{P_{k}(\lambda)}$ is strictly decreasing in $J$.

Let $P_{k-1}(y)=0$ and $(x, z) \subset(0, \infty)$ be an interval that contains $y$, but no zeros of $P_{k}(\lambda)$. Then, $\frac{P_{k+1}(\lambda)}{P_{k}(\lambda)}$ is strictly decreasing in intervals $(x, y)$ and $(y, z)$. Thus, the continuity yields $\frac{P_{k+1}(\lambda)}{P_{k}(\lambda)}$ is strictly decreasing in interval $(x, z)$.

(b) Let $K \subset(0, \infty)$ be an interval that has no zeros of $P_{k}(\lambda)$ and $P_{k+1}(\lambda)$. From the recurrence relation $(2.1)$, we have

$$
\begin{aligned}
{\left[\frac{\lambda P_{k}(\lambda)}{P_{k+1}(\lambda)}\right]^{-1} } & =\frac{P_{k+1}(\lambda)}{\lambda P_{k}(\lambda)}=\frac{a_{k+1}-a_{k}-\lambda}{\lambda}-a_{k} \frac{P_{k-1}(\lambda)}{P_{k}(\lambda)} \\
& =\frac{a_{k+1}-a_{k}}{\lambda}-1-a_{k} \frac{P_{k-1}(\lambda)}{P_{k}(\lambda)} .
\end{aligned}
$$

Since $a_{k+1}>a_{k}, \frac{a_{k+1}-a_{k}}{\lambda}$ is strictly decreasing, and also the continuity and the assumption (for $i \leq k$ ) show that $-a_{k} \frac{P_{k-1}(\lambda)}{P_{k}(\lambda)}$ is strictly decreasing for $a_{k}>0$ in $K$. Therefore, we have $\left[\frac{\lambda P_{k}(\lambda)}{P_{k+1}(\lambda)}\right]^{-1}$ is strictly decreasing and $\frac{\lambda P_{k}(\lambda)}{P_{k+1}(\lambda)}$ is strictly increasing in $K$. Furthermore, even if $K$ contains any zeros of $P_{k}(\lambda)$, then the continuity yields $\frac{\lambda P_{k}(\lambda)}{P_{k+1}(\lambda)}$ is strictly increasing in $K$. This completes the inductive steps and proofs of $(a)$ and $(b)$.

(c) The degree of $P_{i}(\lambda)$ is $i$ and $P_{i}(\lambda)$ is of the form

$$
P_{i}(\lambda)=(-1)^{i} \lambda^{i}+\cdots .
$$

This immediately yields

$$
\lim _{\lambda \rightarrow \infty} \frac{\lambda P_{i-1}(\lambda)}{P_{i}(\lambda)}=-1
$$

and the sign of $P_{i}(\infty)$ is $(-1)^{i}$. In view of $(b)$, we have $\frac{\lambda P_{i-1}(\lambda)}{P_{i}(\lambda)}<-1$ for $\lambda \in I_{3}$. Thus,

$$
\frac{(-1)^{i-1} \lambda P_{i-1}(\lambda)}{(-1)^{i-2} P_{i}(\lambda)}>1
$$

Since $P_{i}(\lambda)$ has no zeros in $I_{3}$, the sign of $P_{i}(\lambda)$ does not change in $I_{3}$. This shows that the sign of $P_{i}(\lambda)$ in $I_{3}$ equals to the sign of $P_{i}(\infty)$. That is,

$$
(-1)^{i} P_{i}(\lambda)>0\left(\text { or }(-1)^{i-2} P_{i}(\lambda)>0\right)
$$

for $\lambda \in I_{3}$. Thus, we have

$$
(-1)^{i-2} P_{i}(\lambda)<(-1)^{i-1} \lambda P_{i-1}(\lambda)
$$

Theorem 2.4. Suppose that the real sequence $\left\{a_{i}\right\}$ is positive and strictly increasing, then the sequence

$$
P_{0}(\lambda)=1, P_{1}(\lambda), P_{2}(\lambda), \ldots, P_{n-1}(\lambda), P_{n}(\lambda)
$$

has the Sturm property on interval $(0, \infty)$.

Proof. We must show that four conditions $(a)-(d)$ in Definition 1.1 are satisfied.

(a) It is clear that $P_{0}(\lambda)=1$ has no zeros. 
(b) $P_{1}(\lambda)=a_{1}-\lambda$ has only one zero as $\lambda_{0}=a_{1}$. Thus, (b) is true for $i=1$.

Assume that $(b)$ is valid for $i \leq k$, then the set of zeros of $P_{k}(\lambda)$ is discrete. By the recurrence relation (2.1), we have

$$
P_{k+1}(\lambda)=P_{k}(\lambda)\left[\left(a_{k+1}-a_{k}-\lambda\right)-a_{k} \lambda \frac{P_{k-1}(\lambda)}{P_{k}(\lambda)}\right] .
$$

From Lemma $2.2(b), P_{k+1}(\lambda)$ and $P_{k}(\lambda)$ have no common zero, and from Lemma $2.3(a), \frac{P_{k+1}(\lambda)}{P_{k}(\lambda)}=\left(a_{k+1}-a_{k}-\lambda\right)-a_{k} \lambda \frac{P_{k-1}(\lambda)}{P_{k}(\lambda)}$ is strictly decreasing between any two consecutive zeros of $P_{k}(\lambda)$. Thus, $P_{k+1}(\lambda)$ has at most one zero between any two consecutive zeros of $P_{k}(\lambda)$. That is, $(b)$ is true for $k+1 \leq n$.

(c) For $1 \leq i \leq n-1$, by the recurrence relation (2.1) we have

$$
P_{i+1}(\lambda)=\left(a_{i+1}-a_{i}-\lambda\right) P_{i}(\lambda)-a_{i} \lambda P_{i-1}(\lambda) .
$$

If $P_{i}(\lambda)=0$ for $1 \leq i \leq n-1$, then $P_{i+1}(\lambda)=-a_{i} \lambda P_{i-1}(\lambda)$, since $a_{i}>0$, $P_{i+1}(\lambda) P_{i-1}(\lambda)<0$ for $\lambda \in(0, \infty)$.

(d) Let $P_{i}\left(\lambda_{0}\right)=0$ for $1 \leq i \leq n$ and $\left[\lambda_{0}-\varepsilon_{1}, \lambda_{0}+\varepsilon_{2}\right]$ be an interval that contains no zeros of $P_{i-1}(\lambda)$ for sufficiently small $\varepsilon_{1}, \varepsilon_{2}>0$. Then, the sign of $P_{i-1}(\lambda)$ does not change and from Lemma $2.3(a), \frac{P_{i}(\lambda)}{P_{i-1}(\lambda)}$ is strictly decreasing in this interval. Thus, the sign of $\frac{P_{i}(\lambda)}{P_{i-1}(\lambda)}$ (or equivalently the sign of $P_{i-1}(\lambda) P_{i}(\lambda)$ ) is $(+)$ and $(-)$ in intervals $\left[\lambda_{0}-\varepsilon_{1}, \lambda_{0}\right)$ and $\left(\lambda_{0}, \lambda_{0}+\varepsilon_{2}\right]$, respectively. That is,

$$
P_{i-1}\left(\lambda_{0}-\varepsilon_{1}\right) P_{i}\left(\lambda_{0}-\varepsilon_{1}\right)>0>P_{i-1}\left(\lambda_{0}+\varepsilon_{2}\right) P_{i}\left(\lambda_{0}+\varepsilon_{2}\right) .
$$

Since the sign of $P_{i-1}(\lambda)$ does not change in interval $\left[\lambda_{0}-\varepsilon_{1}, \lambda_{0}+\varepsilon_{2}\right]$, we have

$$
P_{i-1}\left(\lambda_{0}\right) P_{i}\left(\lambda_{0}-\varepsilon_{1}\right)>0>P_{i-1}\left(\lambda_{0}\right) P_{i}\left(\lambda_{0}+\varepsilon_{2}\right)
$$

and

which completes the proof.

$$
P_{i-1}\left(\lambda_{0}\right)\left[P_{i}\left(\lambda_{0}+\varepsilon_{2}\right)-P_{i}\left(\lambda_{0}-\varepsilon_{1}\right)\right]<0
$$

Theorem 2.5. Suppose that the real sequence $\left\{a_{i}\right\}$ is positive and strictly increasing, then all the eigenvalues of the matrix $\left[F_{a}\right]_{n}$ are different and positive for $n \geq 1$.

Proof. Let $\lambda^{i}$ and $\lambda^{\imath \imath}$ be the smallest and the largest zeros of all $P_{i}(\lambda)$ for $1 \leq i \leq n$, respectively. Since 0 is not a zero of $P_{i}(\lambda)$, the sign of $P_{i}(\lambda)$ does not change in interval $\left[0, \lambda^{\imath}\right)$. Then, the sign of $P_{i}(x)$ is equal to sign of $P_{i}(0)$ for $x \in\left(0, \lambda^{2}\right)$. Thus, we get $w_{i}(x)=w_{i}(0)$, where $w_{i}(\alpha)$ is the number of sign changes of the sequence

$$
P_{0}(\alpha), P_{1}(\alpha), P_{2}(\alpha), \ldots, P_{i-1}(\alpha), P_{i}(\alpha)
$$

for $1 \leq i \leq n$. Similarly, since the sign of $P_{i}(\lambda)$ does not change in interval $\left(\lambda^{\imath \imath}, \infty\right)$ and $w_{i}(y)=w_{i}(\infty)$ for $y \in\left(\lambda^{\imath}, \infty\right)$. By Theorems 1.2 and 2.4, for the number of different zeros of $P_{i}(\lambda)$ in interval $(x, y)$ is $w_{i}(y)-w_{i}(x)$. Since $w_{i}(y)-w_{i}(x)=w_{i}(\infty)-w_{i}(0)$ for $x \in\left(0, \lambda^{\imath}\right)$ and $y \in\left(\lambda^{\imath \imath}, \infty\right)$, we must compute the values $w_{i}(\infty)$ and $w_{i}(0)$.

The degree of $P_{i}(\lambda)$ is $i$ and $P_{i}(\lambda)$ is of the form

$$
P_{i}(\lambda)=(-1)^{i} \lambda^{i}+\cdots \cdot
$$

Then, the sign of $P_{i}(\infty)$ is $(-1)^{i}$. Thus, we have

$$
w_{i}(\infty)=i \text {. }
$$

Since $a_{i}$ is a positive real number and the sequence $\left\{a_{i}\right\}$ is strictly increasing,

$$
P_{i}(0)=\left(a_{i}-a_{i-1}\right)\left(a_{i-1}-a_{i-2}\right) \cdots\left(a_{2}-a_{1}\right) a_{1}>0 .
$$


Then, we have $w_{i}(0)=0$. Thus, the number of different zeros of $P_{i}(\lambda)$ in interval $(x, y)$ for $x \in\left(0, \lambda^{\imath}\right)$ and $y \in\left(\lambda^{\imath \imath}, \infty\right)$ is

$$
w_{i}(y)-w_{i}(x)=w_{i}(\infty)-w_{i}(0)=i .
$$

Since the number of zeros of $P_{i}(\lambda)$ is $i$, all the zeros of $P_{i}(\lambda)$ are in interval $(x, y)$ for $x \in\left(0, \lambda^{\imath}\right)$ and $y \in\left(\lambda^{\imath \imath}, \infty\right)$. Hence, all the zeros of $P_{i}(\lambda)$ are different and positive for $1 \leq i \leq n$. This shows that all the eigenvalues of the matrix $\left[F_{a}\right]_{n}$ are different and positive for $n \geq 1$.

Theorem 2.6. Suppose that the real sequence $\left\{a_{i}\right\}$ is positive and strictly increasing, then for $1 \leq i \leq n$, the eigenvalues of $\left[F_{a}\right]_{i}$ and $\left[F_{a}\right]_{i-1}$ are interlaced. That is,

$$
\lambda_{1}^{(i)}>\lambda_{1}^{(i-1)}>\lambda_{2}^{(i)}>\lambda_{2}^{(i-1)}>\cdots>\lambda_{i-1}^{(i-1)}>\lambda_{i}^{(i)},
$$

where $\lambda_{s}^{(i)}$ is an eigenvalue of $\left[F_{a}\right]_{i}$ for $s=1,2, \ldots, i$.

Proof. Theorems 1.3 and 2.4 give the claimed result immediately.

Corollary 2.7. Suppose that the real sequence $\left\{a_{i}\right\}$ is positive and strictly increasing, then

(i) $\lambda_{n}<a_{1}$,

(ii) $a_{n}<\lambda_{1}$,

where $\lambda_{n}$ and $\lambda_{1}$ are the smallest and the largest eigenvalues of $\left[F_{a}\right]_{n}$ for $n \geq 2$, respectively.

Proof. (i) This follows from Theorem 2.6, since $a_{1}$ is unique eigenvalue of $\left[F_{a}\right]_{1}$.

(ii) Let $\mu_{1}$ be the largest eigenvalue of $\left[F_{a}\right]_{n-1}$ (or equavalently $\mu_{1}$ be the largest zero of $P_{n-1}(\lambda)$ ). Then, $\mu_{1}$ is greater then all the zeros of all $P_{k}(\lambda)$ and the sign of $P_{k}(\lambda)$ is $(-1)^{k}$ in interval $\left(\mu_{1}, \infty\right)$ for $0 \leq k \leq n-1$.

If $a_{n}>\mu_{1}$, then the sign of $P_{k}\left(a_{n}\right)$ is $(-1)^{k}$ for $0 \leq k \leq n-1$. This shows that $w_{n-1}\left(a_{n}\right)=n-1$. From the recurrence relation (2.1), we have

$$
\begin{aligned}
P_{n}\left(a_{n}\right) & =-a_{n-1} P_{n-1}\left(a_{n}\right)-a_{n-1} a_{n} P_{n-2}\left(a_{n}\right) \\
& =-a_{n-1}\left[P_{n-1}\left(a_{n}\right)+a_{n} P_{n-2}\left(a_{n}\right)\right] .
\end{aligned}
$$

By Lemma $2.3(c)$, we have

$$
\begin{gathered}
(-1)^{n-3} P_{n-1}\left(a_{n}\right)<(-1)^{n-2} a_{n} P_{n-2}\left(a_{n}\right), \\
(-1)^{n-3}\left[P_{n-1}\left(a_{n}\right)+a_{n} P_{n-2}\left(a_{n}\right)\right]<0
\end{gathered}
$$

and the sign of $P_{n-1}\left(a_{n}\right)+a_{n} P_{n-2}\left(a_{n}\right)$ is $(-1)^{n-2}$. Then, by equation (2.6), the sign of $P_{n}\left(a_{n}\right)$ is $(-1)^{n-1}$. That is, $P_{n}\left(a_{n}\right)$ and $P_{n-1}\left(a_{n}\right)$ have the same sign. Thus,

$$
w_{n}\left(a_{n}\right)=w_{n-1}\left(a_{n}\right)=n-1 .
$$

Moreover, $w_{n}(\infty)=n$ and $P_{n}\left(a_{n}\right) \neq 0$. Then, Theorems 1.2 and 2.4 yield $P_{n}(\lambda)$ has $w_{n}(\infty)-w_{n}\left(a_{n}\right)=n-(n-1)=1$ real zero (or equavalently $\left[F_{a}\right]_{n}$ has one eigenvalue) in interval $\left(a_{n}, \infty\right)$. Thus, $a_{n}<\lambda_{1}$.

If $a_{n} \leq \mu_{1}$, then from Theorem 2.6, $\mu_{1}<\lambda_{1}$. Hence, $a_{n}<\lambda_{1}$.

Remark 2.8. Our results work even if the real sequence $\left\{a_{i}\right\}$ is negative and strictly decreasing. For example, if $b_{i}=-a_{i}$, then $b_{i}$ is a positive real number, the sequence $\left\{b_{i}\right\}$ is strictly increasing and $\left[F_{a}\right]_{n}=-\left[F_{b}\right]_{n}$. Since all the eigenvalues of $\left[F_{b}\right]_{n}$ are positive, all the eigenvalues of $\left[F_{a}\right]_{n}$ are negative. 


\section{An example}

In this section, we illustrate our results with the following example.

Example 3.1. Consider the finite sequence $a=(2,4,6,8,10)$. Then, $a_{i \leq 5}=2 i$ and the corresponding generalized Frank matrix to the sequence $a=(2,4,6,8,10)$ is

$$
\left[F_{a}\right]_{5}=\left[\begin{array}{ccccc}
10 & 8 & 0 & 0 & 0 \\
8 & 8 & 6 & 0 & 0 \\
6 & 6 & 6 & 4 & 0 \\
4 & 4 & 4 & 4 & 2 \\
2 & 2 & 2 & 2 & 2
\end{array}\right] .
$$

For the characteristic polynomials of $\left[F_{a}\right]_{i \leq 5}$, Lemma 2.1 yields for $i \geq 2$

$$
P_{i}(\lambda)=(2-\lambda) P_{i-1}(\lambda)-a_{i-1} \lambda P_{i-2}(\lambda) .
$$

Thus,

$$
\begin{aligned}
P_{0}(\lambda) & =1 \\
P_{1}(\lambda) & =2-\lambda \\
P_{2}(\lambda) & =(2-\lambda)(2-\lambda)-2 \lambda=\lambda^{2}-6 \lambda+4 \\
P_{3}(\lambda) & =(2-\lambda)\left(\lambda^{2}-6 \lambda+4\right)-4 \lambda(2-\lambda)=-\lambda^{3}+12 \lambda^{2}-24 \lambda+8 \\
P_{4}(\lambda) & =(2-\lambda)\left(-\lambda^{3}+12 \lambda^{2}-24 \lambda+8\right)-6 \lambda\left(\lambda^{2}-6 \lambda+4\right) \\
& =\lambda^{4}-20 \lambda^{3}+84 \lambda^{2}-80 \lambda+16 \\
P_{5}(\lambda) & =(2-\lambda)\left(\lambda^{4}-20 \lambda^{3}+84 \lambda^{2}-80 \lambda+16\right)-8 \lambda\left(-\lambda^{3}+12 \lambda^{2}-24 \lambda+8\right) \\
& =-\lambda^{5}+30 \lambda^{4}-220 \lambda^{3}+440 \lambda^{2}-240 \lambda+32 .
\end{aligned}
$$

If we compute $P_{i \leq 5}(\lambda)$ using $\operatorname{det}\left(\left[F_{a}\right]_{i \leq 5}-\lambda I\right)$, then we obtain the same results as above. Furthermore, the roots of $P_{i \leq 5}(\lambda)$ (or the eigenvalues of $\left[F_{a}\right]_{i \leq 5}$ ) are

$$
\begin{gathered}
\lambda_{1}^{(1)}=2, \\
\lambda_{1}^{(2)}=5,2360, \lambda_{2}^{(2)}=0.7639, \\
\lambda_{1}^{(3)}=9.5825, \lambda_{2}^{(3)}=2, \lambda_{3}^{(3)}=0.4174, \\
\lambda_{1}^{(4)}=14.6254, \lambda_{2}^{(4)}=4.1332, \lambda_{3}^{(4)}=0.9677, \lambda_{4}^{(4)}=0.2734, \\
\lambda_{1}^{(5)}=20.1258, \lambda_{2}^{(5)}=7.1131, \lambda_{3}^{(5)}=2, \lambda_{4}^{(5)}=0.5623, \lambda_{5}^{(5)}=0.1987,
\end{gathered}
$$

where $\lambda_{s}^{(i)}$ denotes the roots of $P_{i \leq 5}(\lambda)$ for $s=1,2, \ldots, i$. Hence, we observe:

- $P_{i \leq 5}(\lambda)$ does not vanish for $\lambda=0$,

- $P_{i \leq 4}(\lambda)$ and $P_{i+1}(\lambda)$ have not a common zero,

- The set of zeros of $P_{i \leq 5}(\lambda)$ is discrete,

- If $P_{i \leq 4}(\lambda)=0$, then $P_{i-1}(\lambda) P_{i+1}(\lambda)<0$. For example, since $P_{2}(2)=-4$, $P_{3}(2)=0, P_{4}(2)=48$, we have $P_{2}(2) \cdot P_{4}(2)=-192<0$,

- If $P_{i}\left(\lambda_{0}\right)=0$, then for sufficiently small $\varepsilon_{1}, \varepsilon_{2}>0$,

$$
P_{i-1}\left(\lambda_{0}\right)\left[P_{i}\left(\lambda_{0}+\varepsilon_{2}\right)-P_{i}\left(\lambda_{0}-\varepsilon_{1}\right)\right]<0,
$$

where $1 \leq i \leq 5$. For example, since

$P_{3}(2)=0, P_{2}(2)=-4, P_{3}\left(2+\frac{1}{1000}\right)=0.0120, P_{3}\left(2-\frac{1}{100}\right)=-0.1193$,

we have

$$
P_{2}(2)\left[P_{3}\left(2+\frac{1}{1000}\right)-P_{3}\left(2-\frac{1}{100}\right)\right]=-0.5252<0,
$$

where $\varepsilon_{1}=\frac{1}{100}, \varepsilon_{2}=\frac{1}{1000}$,

- The sequence $P_{0}(\lambda), P_{1}(\lambda), P_{2}(\lambda), P_{3}(\lambda), P_{4}(\lambda)$ and $P_{5}(\lambda)$ is a Sturm sequence, 
- The eigenvalues of $\left[F_{a}\right]_{i \leq 5}$ (or the zeros of $P_{i \leq 5}(\lambda)$ ) are positive and different,

- The eigenvalues of $\left[F_{a}\right]_{i \leq 5}$ and $\left[F_{a}\right]_{i-1}$ are interlaced. For example,

$$
\begin{aligned}
& \lambda_{1}^{(5)}=20.1258>\lambda_{1}^{(4)}=14.6254>\lambda_{2}^{(5)}=7.1131>\lambda_{2}^{(4)}=4.1332> \\
& \lambda_{3}^{(5)}=2>\lambda_{3}^{(4)}=0.9677>\lambda_{4}^{(5)}=0.5623>\lambda_{4}^{(4)}=0.2734>\lambda_{5}^{(5)}=0.1987 \text {, } \\
& \text { - } \lambda_{1}^{(2)}=5.2360>a_{2}=4, \lambda_{1}^{(3)}=9.5825>a_{3}=6, \lambda_{1}^{(4)}=14.6254>a_{4}=8 \text {, } \\
& \lambda_{1}^{(5)}=20.1258>a_{5}=10 \\
& \lambda_{2}^{(2)}=0.7639<a_{1}=2, \lambda_{3}^{(3)}=0.4174<a_{1}=2, \lambda_{4}^{(4)}=0.2734<a_{1}=2 \text {, } \\
& \lambda_{5}^{(5)}=0.1987<a_{1}=2 \text {, }
\end{aligned}
$$

as stated in the results in the previous section.

Finally, we compute the numbers of eigenvalues of $\left[F_{a}\right]_{5}$ in intervals $(0,3)$ and $(3,25)$. So then, we need the number of sign changes of $P_{i \leq 5}(\lambda)$ for $\lambda=0, \lambda=3$ and $\lambda=25$. Table 1 serves this need. From Table 1 , we have $w_{5}(0)=0, w_{5}(3)=3$ and $w_{5}(25)=5$, where $w_{5}(\alpha)$ denotes the number of changes in sign of $P_{i \leq 5}(\alpha)$. Thus, the number of eigenvalues of $\left[F_{a}\right]_{5}$ in interval $(0,3)$ is $w_{5}(3)-w_{5}(0)=3-0=3$ and the number of eigenvalues of $\left[F_{a}\right]_{5}$ in interval $(3,25)$ is $w_{5}(25)-w_{5}(3)=5-3=2$. Really, the eigenvalues of $\left[F_{a}\right]_{5}$ are $20.1258,7.1131,2,0.5623$ and 0.1987 .

\begin{tabular}{|l|c|c|c|}
\hline & $\begin{array}{c}\text { Sign of } P_{i}(\lambda) \\
\text { for } \lambda=0\end{array}$ & $\begin{array}{c}\text { Sign of } P_{i}(\lambda) \\
\text { for } \lambda=3\end{array}$ & $\begin{array}{c}\text { Sign of } P_{i}(\lambda) \\
\text { for } \lambda=25\end{array}$ \\
\hline$P_{0}(\lambda)=1$ & + & + & + \\
$P_{1}(\lambda)=2-\lambda$ & + & - & - \\
$P_{2}(\lambda)=\lambda^{2}-6 \lambda+4$ & + & - & + \\
$P_{3}(\lambda)=-\lambda^{3}+12 \lambda^{2}-24 \lambda+8$ & + & + & - \\
$P_{4}(\lambda)=\lambda^{4}-20 \lambda^{3}+84 \lambda^{2}-80 \lambda+16$ & + & + & + \\
$P_{5}(\lambda)=-\lambda^{5}+30 \lambda^{4}-220 \lambda^{3}+440 \lambda^{2}-240 \lambda+32$ & + & - & - \\
\hline Number of sign changes & $w_{5}(0)=0$ & $w_{5}(3)=3$ & $w_{5}(25)=5$ \\
\hline
\end{tabular}

Table 1. The number of sign changes of $P_{i \leq 5}(\lambda)$ for $\lambda=0, \lambda=3$ and $\lambda=25$.

\section{References}

[1] P.G. Ciarlet and J.L. Lions, Handbook of numerical analysis, Vol. III: Solution of equations in $\mathbb{R}^{n}$ (Part 2), 625-778, Elsevier, Amsterdam, 1994.

[2] P.J. Eberlein, A note on the matrices denoted $B_{n}^{*}$, SIAM J. Appl. Math. 20 (1), 87-92, 1971.

[3] W.L. Frank, Computing eigenvalues of complex matrices by determinant evaluation and by methods of Danilewski and Wielandt, J. Soc. Ind. Appl. Math. 6 (4), 378-392, 1958.

[4] L. Greenberg, Sturm sequences for nonlinear eigenvalue problems, SIAM J. Math. Anal. 20 (1), 182-199, 1989.

[5] J.-F. Hake, A remark on Frank matrices, Computing (Wien. Print) 35, 375-379, 1985.

[6] E. Isaacson and H.B. Keller, Analysis of numerical methods, 2nd edition, John Wiley, New York, 1966.

[7] M.K. Jain, S.R.K. Iyengar and R.K. Jain, Numerical methods: problems and solutions, Revised Second Edition, New Age International Publishers, New Delhi, 2004.

[8] E. Kılıç and T. Arıkan, Studying new generalizations of Max-Min matrices with a novel approach, Turkish J. Math. 43, 2010-2024, 2019. 
[9] E.Ö. Mersin, M. Bahşi and A.D. Maden, Some properties of generalized Frank matrices, Mathematical Sciences and Applications E-Notes 8 (2), 170-177, 2020.

[10] A. Mostowski and M. Stark, Introduction to higher algebra, Pergamon Press, 1964.

[11] J.M. Ortega, On Sturm sequences for tridiagonal matrices, J. Assoc. Comput. Mach. 7 (3), 260-263, 1960.

[12] J. Stoer and R. Bulirsch, Introduction to numerical analysis, Springer-Verlag, New York, 1980.

[13] C. Sturm, Analyse d' un Mémoire sur la résolution des équations numériques, Collected Works of Charles François Sturm, Birkhäuser Basel, 323-326, 2009.

[14] C. Sturm, Extrait d'un Mémoire sur L'intécration d'un système d'équations différentielles linéaires, présenté à l'Académie des sciences, Collected Works of Charles François Sturm, Birkhäuser Basel, 334-342, 2009.

[15] C. Sturm, Mémoire sur la résolution des équations numériques, Collected Works of Charles François Sturm, Birkhäuser Basel, 345-390, 2009.

[16] J.M. Varah, A generalization of the Frank matrix, SIAM J. Sci. and Stat. Comput. 7 (3), 835-839, 1986.

[17] J.H. Wilkinson, The algebraic eigenvalue problem, Oxford University Press, 1965. 